\title{
Measuring adolescents' exposure to victimization: The Environmental Risk (E-Risk) Longitudinal Twin Study
}

\author{
HELEN L. FISHER,${ }^{a}$ AVSHALOM CASPI,${ }^{a, b}$ TERRIE E. MOFFITT,${ }^{a, b}$ JASMIN WERTZ,${ }^{a}$ REBECCA GRAY,${ }^{a}$ \\ JOANNE NEWBURY, ${ }^{a}$ ANTONY AMBLER,${ }^{a}$ HELENA ZAVOS,${ }^{a}$ ANDREA DANESE,${ }^{a, c}$ JONATHAN MILL,${ }^{a, d}$

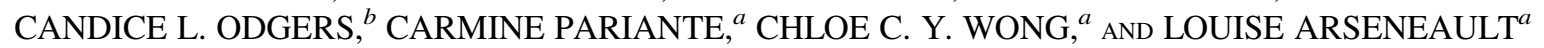 \\ ${ }^{a}$ King's College London; ${ }^{b}$ Duke University; ${ }^{c}$ South London \& Maudsley NHS Foundation Trust; and ${ }^{d}$ University of Exeter \\ Medical School
}

\begin{abstract}
This paper presents multilevel findings on adolescents' victimization exposure from a large longitudinal cohort of twins. Data were obtained from the Environmental Risk (E-Risk) Longitudinal Twin Study, an epidemiological study of 2,232 children (1,116 twin pairs) followed to 18 years of age (with 93\% retention). To assess adolescent victimization, we combined best practices in survey research on victimization with optimal approaches to measuring life stress and traumatic experiences, and introduce a reliable system for coding severity of victimization. One in three children experienced at least one type of severe victimization during adolescence (crime victimization, peer/sibling victimization, Internet/mobile phone victimization, sexual victimization, family violence, maltreatment, or neglect), and most types of victimization were more prevalent among children from low socioeconomic backgrounds. Exposure to multiple victimization types was common, as was revictimization; over half of those physically maltreated in childhood were also exposed to severe physical violence in adolescence. Biometric twin analyses revealed that environmental factors had the greatest influence on most types of victimization, while severe physical maltreatment from caregivers during adolescence was predominantly influenced by heritable factors. The findings from this study showcase how distinct levels of victimization measurement can be harmonized in large-scale studies of health and development.
\end{abstract}

Children exposed to various forms of victimization during childhood have repeatedly been shown to have a range of adverse physical, social, cognitive, and mental health outcomes (Gilbert et al., 2009), and we are now beginning to appreciate that such early life stressors have implications for health and well-being across the life span (Cicchetti \& Tucker, 1994; Currie \& Widom, 2010; Danese et al., 2009; Moffitt \& the Klaus Grawe ThinkTank, 2013; Norman et al., 2012; Takizawa, Maughan, \& Arseneault, 2014; Widom, Czaja, Bentley, \& Johnson, 2012). Although much research has focused on victimization exposure during the early stages of childhood, adolescence is also a time of major emotional, physical, social, and neurodevelopmental change (Cromer, 2011; Luci-

We are grateful to the study mothers and fathers, the twins, and the twins' teachers for their participation. Our thanks to members of the Environmental Risk (ERisk) Longitudinal Twin study team for their dedication, hard work, and insights; to Renate Houts for statistical assistance; and to Benjamin Bleiberg, Perry Dinardo, and Stephanie Gandleman for their research assistance. The E-Risk Study is funded by the Medical Research Council (UKMRC) Grant G1002190. Additional support was provided by National Institute of Child Health and Development Grant HD077482, Economic and Social Research Council Grant RES-177-25-0013, the Jacobs Foundation, UKMRC Population Health Scientist fellowship Grant G1002366 (to H.L.F.), and MQ Fellows Award MQ14F40 (to H.L.F.).

Address correspondence and reprint requests to: Louise Arseneault, MRC SGDP Centre, Institute of Psychiatry, Psychology \& Neuroscience, King's College London, 16 De Crespigny Park, London SE5 8AF, UK; E-mail: louise.ar seneault@kcl.ac.uk. ana, 2013), suggesting that victimization during this period could have equally important ramifications for development. Moreover, as adolescents spend an increasing proportion of their time outside of the home environment, compared to when they were children, they are also likely to experience a greater variety of victimization exposures. Thus, it is important to measure exposure to a range of possible victimization experiences during this key transition period and examine their immediate and long-term consequences in affected individuals.

However, there is no current consensus about the optimal method for assessing exposure to such victimization experiences. Reports obtained from social services and medical or police records are advocated to be the most objective source of information on victimization exposure (Widom, 1988). However, these capture only a fraction of victimization cases because most cases do not come to the attention of such services (Widom, Czaja, \& DuMont, 2015), and those that do may overrepresent children from poorer backgrounds (Pelton, 1978) or more extreme cases of physical abuse or neglect (Groeneveld \& Giovannoni, 1977). Therefore, in order to obtain more complete rates of victimization, it is necessary to question individuals directly. There is an ongoing tension in research utilizing self-report measures of victimization between employing self-report checklists or questionnaires, or more comprehensive interview measures (Monroe, 2008). Interview-based assessments of stressful or traumatic events are considered to be superior to self-report checklists or 
structured questionnaires (Brown, 1989; Dohrenwend, 2006; Gorman, 1993; Monroe, 2008; Paykel, 2001), because interviews are believed to be less influenced by respondent bias and subjective interpretation of questionnaire items (Dohrenwend, 2006; Grant, Compas, Thurm, McMahon, \& Gipson, 2004; Hepp et al., 2006; Monroe \& McQuaid, 1994), thus providing more precision and reliability when measuring relevant exposures. Nonetheless, published research into childhood and adolescent stress exposure has predominantly utilized self-report checklists or questionnaires (Grant et al., 2004). Resistance to using interviews has mainly revolved around the considerable time and resources required to administer these forms of assessment (Dohrenwend, 2006). Thus, interview-based measures have often been considered unfeasible for large-scale epidemiological studies. Hence, in order for the field to move forward, a compromise needs to be reached between these two levels of measurement: self-report questionnaires and checklists derived from large-sample survey methodology, with their greater cost-effectiveness and scalability; and interview-based measures of victimization derived from small-sample clinical methodology, with their more in-depth coverage and investigator-based rating systems.

In this paper, we describe adolescent victimization in a large-scale epidemiological twin study using a method that combined a standardized survey with a more in-depth contextual coding system. Specifically, we started by adapting the Juvenile Victimization Questionnaire (JVQ; Finkelhor, Hamby, Turner, \& Ormrod, 2011; Hamby, Finkelhor, Ormrod, \& Turner, 2004) as a clinical interview. The JVQ is a standardized questionnaire that has been used to obtain a wide range of self-reported victimization experiences from large samples of adolescents in both the United Kingdom (Radford, Corral, Bradley, \& Fisher, 2013) and the United States (Finkelhor, Hamby, Ormrod, \& Turner, 2005). However, the data derived from the JVQ are essentially a count of the items the respondent endorses and may lack sufficient detail to determine the severity of victimization experienced or to evaluate the context in which the victimization occurred. These missing features are considered to be desirable to grasp the fuller picture of victimization exposure (Barnett, Manly, \& Cicchetti, 1993; Brown, 1974; Brown \& Harris, 1978). Severity of exposure, in particular, has been shown to predict later psychopathology (Clemmons, Walsh, DiLillo, \& Messman-Moore, 2007; Fergusson, Boden, \& Horwood, 2008; Schilling, Aseltine, \& Gore, 2008). Therefore, we utilized the existing questions from the JVQ but administered these as part of an interview in which respondents provided detailed descriptions of their victimization experiences. These descriptions were coded by an independent panel of expert raters using a coding system adapted from the Childhood Experience of Care and Abuse Interview (Bifulco, Brown, \& Harris, 1994; Bifulco, Brown, Neubauer, Moran, \& Harris, 1994), which provides standardized anchor points for determining severity of exposure within the relevant context. It is hoped that our pragmatic approach to blending two leading traditions in the assessment of victimization will enable future researchers to more fully explore the role of multiple types of victimization in the etiology of physical and mental health.

This paper has two main aims. First, we detail this combined approach to assessing adolescent victimization and present the reliability of its implementation in a longitudinal cohort of twin children. Second, we report initial findings concerning the prevalence of different types of severe victimization using this combined assessment method, along with their co-occurrence (polyvictimization), and recurrence from childhood to adolescence (revictimization), and we examine how demographic factors influence variability in exposure. We also exploit the twin design of our study to explore the relative genetic and environmental influences on exposure to each type of adolescent victimization experience.

\section{Method}

\section{Study cohort}

Participants were members of the Environmental Risk (ERisk) Longitudinal Twin Study, which tracks the development of a birth cohort of 2,232 British children. The sample was drawn from a larger birth register of twins born in England and Wales in 1994-1995 (Trouton, Spinath, \& Plomin, 2002). Full details about the sample are reported elsewhere (Moffitt \& the E-Risk Study Team, 2002). Briefly, the ERisk sample was constructed in 1999-2000, when 1,116 families (93\% of those eligible) with same-sex 5-year-old twins participated in home-visit assessments. This sample comprised 55\% monozygotic (MZ) and 45\% dizygotic (DZ) twin pairs; sex was evenly distributed within zygosity ( $49 \%$ male). Families were recruited to represent the UK population of families with newborns in the 1990s, on the basis of residential location throughout England and Wales and mother's age. Teenaged mothers with twins were overselected to replace high-risk families who were selectively lost to the register through nonresponse. Older mothers having twins via assisted reproduction were underselected to avoid an excess of well-educated older mothers.

At follow-up, the study sample represents the full range of socioeconomic conditions in the United Kingdom, as reflected in the families' distribution on a neighborhood-level socioeconomic index (A Classification of Residential Neighbourhoods [ACORN], developed by CACI Inc. for commercial use in Great Britain; Odgers, Caspi, Russell, et al., 2012). ACORN uses census and other survey-based geodemographic discriminators to classify enumeration districts $(\sim 150$ households) into socioeconomic groups ranging from "wealthy achievers" (Category 1), with high incomes, large single-family houses, and access to many amenities, to "hard-pressed" neighborhoods (Category 5), dominated by government-subsidized housing estates, low incomes, high unemployment, and single parents. ACORN classifications were geocoded to match the location of each E-Risk study family's home (Odgers, Caspi, Bates, Sampson, \& 
Moffitt, 2012). E-Risk families' ACORN distribution closely matches that of households nationwide: $25.6 \%$ of E-Risk families live in "wealthy achiever" neighborhoods compared to $25.3 \%$ nationwide; $5.3 \%$ versus $11.6 \%$ live in "urban prosperity" neighborhoods; $29.6 \%$ versus $26.9 \%$ live in "comfortably off" neighborhoods; $13.4 \%$ versus $13.9 \%$ live in "moderate means" neighborhoods; and $26.1 \%$ versus $20.7 \%$ live in "hard-pressed" neighborhoods. E-Risk underrepresents "urban prosperity" neighborhoods because such households are likely to be childless.

Follow-up home visits were conducted when the children were aged 7 (98\% participation), 10 (96\% participation), 12 (96\% participation), and, most recently in 2012-2014, 18 years (93\% participation). There were 2,066 children who participated in the E-Risk assessments at age 18, and the proportions of MZ (55\%) and male same-sex (47\%) twins were almost identical to those found in the original sample at age 5 . The average age of the twins at the time of the assessment was 18.4 years $(S D=0.36)$; all interviews were conducted after the 18th birthday. There were no differences between those who did and did not take part at age 18 in terms of socioeconomic status (SES) assessed when the cohort was initially defined $\left(\chi^{2}=0.86, p=.65\right)$, age 5 IQ scores $(t=0.98$, $p=.33$ ), or age 5 internalizing or externalizing behavior problems $(t=0.40, p=.69$ and $t=0.41, p=.68$, respectively). Home visits at ages 5, 7, 10, and 12 years included assessments with participants as well as their mother (or primary caretaker); the home visit at age 18 included interviews only with the participants. Each twin participant was assessed by a different interviewer.

The Joint South London and Maudsley and the Institute of Psychiatry Research Ethics Committee approved each phase of the study. Parents gave informed consent and twins gave assent between 5 and 12 years and then informed consent at age 18 .

\section{Assessment of victimization exposure between ages 12 and 18}

$J V Q$ interview. At age 18, participants were interviewed faceto-face about exposure to a range of adverse experiences between 12 and 18 years using the JVQ (Finkelhor et al., 2011; Hamby et al., 2004), adapted as a clinical interview. The JVQ has good psychometric properties (Finkelhor et al., 2005) and was used in the United Kingdom National Society for the Prevention of Cruelty to Children (NSPCC) national survey (Radford et al., 2011, 2013), thereby providing important benchmark values for comparisons with our cohort. Our adapted JVQ comprised 45 questions covering different forms of victimization grouped into seven categories: crime victimization, peer/sibling victimization, Internet/mobile phone victimization, sexual victimization, family violence, maltreatment, and neglect. The interview schedule used in this study is provided in supplementary materials Part I.

Within each pair of twins in our cohort, cotwins were interviewed separately by a different interviewers and were as- sured of the confidentiality of their responses. The participants were advised that confidentiality would only be broken if they told the interviewers that they were in immediate danger of being hurt, and in such situations, the project leader would be informed and would contact the participant to discuss a plan for safety.

Each JVQ question was asked for the period "since you were 12." Age 12 is a salient age for our participants because it is the age when British children leave primary school to enter secondary school. Participants were given the option to say "yes" or "no" as to whether each type of victimization had occurred in the reporting period. Interviewers could rate each item "maybe" if the participant seemed unsure or hesitant in his or her response or the interviewer was not convinced that the participant understood the question or was paying attention. Items rated as "maybe" were recoded as "no" or "yes" by the rating team based on the notes provided by the interviewers. When insufficient notes were available, then these responses were recoded conservatively as a "no." Consistent with the JVQ manual (Finkelhor et al., 2011; Hamby et al., 2004), participants were coded as 1 if they reported any experience within each type of victimization category or 0 if none of the experiences within the category were endorsed. If an experience was endorsed within a victimization category, follow-up questions were asked concerning how old the participant was when it (first) happened, whether the participant was physically injured in the event, whether the participant was upset or distressed by the event, and how long it went on for (by marking the number of years on a Life History Calendar; Caspi et al., 1996). In addition, the interviewer wrote detailed notes based on the participant's description of the worst event. If multiple experiences were endorsed within a victimization category, the participant was asked to identify and report about his or her worst experience.

Victimization dossiers. All information from the JVQ interview was compiled into victimization dossiers. Using these dossiers, each of the seven victimization categories was rated by an expert in victimology (Dr. Helen Fisher) and 3 other members of the E-Risk team who were trained on using the rating criteria. Ratings were made using a 6-point scale: $0=$ not exposed, then $1-5$ for increasing levels of severity. The anchor points for these ratings were adapted from the coding system used for the Childhood Experience of Care and Abuse interview (CECA; Bifulco, Brown, \& Harris, 1994; Bifulco, Brown, Neubauer, et al., 1994), which has good interrater reliability (Bifulco, Brown, \& Harris, 1994; Bifulco, Brown, Lillie, \& Jarvis, 1997). The CECA is a comprehensive semistructured interview whose standardized coding system attempts to improve the objectivity of ratings by basing them on the coder's perspective (rather than relying on the participant's judgment) and focusing on concrete descriptions rather than perceptions or emotional responses to the questions, together with considering the context in which the adverse experience occurred. This standardization of 
victimization definitions and severity ratings is also crucial to ensure consistency across research groups and disciplines to improve the comparability of findings from different studies (Barnett et al., 1993).

In our adapted coding scheme, the anchor points of the scale differ for each victimization category, with some focused more on the severity of physical injury that is likely to have been incurred during victimization exposure (crime victimization, family violence, and maltreatment), while others are more focused on the frequency of occurrence of victimization (peer/sibling victimization and Internet/mobile phone victimization), the physical intrusiveness of the event (sexual victimization), or the pervasiveness of the effects of victimization (neglect). This reflects the different ways in which severity has previously been defined for different types of victimization (Barnett et al., 1993; Bifulco, Brown, \& Harris, 1994). (Given that our sample comprises twins, we also coded if any of the victimization events experienced by each twin had been perpetrated by their cotwin because it is possible that growing up with a genetically related, sameage child could increase or decrease sibling victimization rates.) Finally, we evaluated whether each participant was exposed to any physical violence, whether in the family, by peers, or by people in the wider environment, based on the entire dossier of victimization experiences. This "any physical violence" exposure variable was also rated on a 6-point scale: $0=$ not exposed, then 1-5 for increasing levels of severity, with severity linked to frequency of occurrence of noninjurious physical attacks at the lower end of the scale (1-3) and the likelihood of incurring an injury and the seriousness of this injury indicating severity at the upper end of the scale (4-5). This rating included violence directed toward the participants themselves as well as violence they witnessed between other people. A copy of our coding scheme is provided in supplementary materials Part II. Each twin's dossier was evaluated separately, and we did not use information provided in the cotwin's dossier about his or her own or shared victimization experiences to rate direct or witnessed violence exposure for the target twin.

Reliability. The first 26 victimization dossiers were coded by all raters to provide training on the use of the severity rating scales and to develop consistency in the application of the anchor points to real-life experiences. Rating discrepancies were discussed by the group and consensus ratings agreed upon. Interrater reliability was conducted on the next 90 dossiers (approximately $4 \%$ of the total sample) with four raters independently scoring all of these dossiers. High levels of interrater reliability were achieved for the severity ratings for all forms of victimization: crime victimization (intraclass correlation coefficient $[\mathrm{ICC}]=0.89, p<.001)$, peer/sibling victimization ( $\mathrm{ICC}=0.91, p<.001)$, Internet/mobile phone victimization ( $\mathrm{ICC}=0.90, p<.001$ ), sexual victimization $(\mathrm{ICC}=0.87, p<0.001)$, family violence $(\mathrm{ICC}=0.93$, $p<0.001$ ), maltreatment (ICC $=0.90, p<.001$ ), neglect (ICC $=0.74, p<.001)$, and any physical violence exposure
(ICC $=0.82, p<.001)$. The remaining dossiers were divided between the four raters in a series of batches of around 100 each, and the raters met at the end of rating each batch to discuss difficult ratings and to reach consensus about any uncertain cases. A random selection of 20 dossiers from each batch of 400 rated ( 5 per rater per batch) was independently rated by a psychologist with expertise in interviewing children and adolescents. Any discrepancies of two points or more on any of the scales were discussed with the rating team and a consensus reached about the final ratings. In addition, all dossiers with ratings of 4 or 5 for any physical violence exposure were independently reviewed by a child psychologist and any discrepancies discussed with the main rating team and consensus reached about the final any physical violence exposure rating.

For reporting purposes in this article, the ratings for each type of victimization were then grouped into three classes: $0=$ no exposure (score of 0$), 1=$ some exposure (score of 1,2 , or 3 ), and $2=$ severe exposure (score of 4 or 5 ) due to small numbers for some of the rating points. Combining ratings of 4 and 5 is also consistent with previous studies using the CECA, which have collapsed comparable scale values to indicate presence of "severe" abuse (e.g., Bifulco, Brown, \& Harris, 1994; Bifulco et al., 1997; Bifulco, Brown, Moran, Ball, \& Campbell, 1998; Fisher, Bunn, Jacobs, Moran, \& Bifulco, 2011).

\section{Childhood SES}

Participants' family SES was defined using a standardized composite of parents' income, education, and social class ascertained at childhood phases of the study, which loaded significantly onto one latent factor (Trzesniewski, Moffitt, Caspi, Taylor, \& Maughan, 2006). The latent factor was divided in tertiles. Thus, of participants who were interviewed at age $18,33 \%$ were characterized as living in a low-SES situation during childhood.

\section{Measures of victimization exposure up to age 12}

Exposure to several types of victimization was assessed repeatedly when the children were $5,7,10$, and 12 years of age, and dossiers have been compiled for each child with cumulative information about exposure to domestic violence between the mother and her partner; frequent bullying by peers; physical maltreatment by an adult (including sexual abuse); and physical neglect. The E-Risk team has previously reported evidence on the reliability and validity of the measures of domestic violence (Moffitt et al., 1997), bullying (Arseneault et al., 2006; Shakoor et al., 2011), and physical maltreatment (Jaffee, Caspi, Moffitt, Polo-Tomas, et al., 2004; Jaffee, Caspi, Moffitt, Polo-Tomas, \& Taylor, 2007), and all the measures are outlined briefly below.

Physical domestic violence. Mothers reported about perpetration of and victimization involving 12 forms of physical vio- 
lence (e.g., slapping, hitting, kicking, and strangling) from the Conflict Tactics Scale (Straus, 1990), on three assessment occasions during the child's first decade of life (when the children were 5, 7, and 10 years of age). Reports of either perpetration or victimization constituted evidence of physical domestic violence. Families in which no physical violence took place were coded as 0 (55.2\%); families in which physical violence took place on one occasion were coded as 1 (28.0\%); and families in which physical violence took place on multiple occasions were coded as $2(16.8 \%)$.

Bullying by peers. Experiences of victimization by bullies were assessed using both mothers' and children's reports. During the interview, the following standard definition of bullying was read out:

Someone is being bullied when another child (a) says mean and hurtful things, makes fun, or calls a person mean and hurtful names; (b) completely ignores or excludes someone from their group of friends or leaves them out on purpose; (c) hits, kicks, or shoves a person, or locks them in a room; (d) tells lies or spreads rumors about them; and (e) other hurtful things like these. We call it bullying when these things happen often, and when it is difficult to make it stop. We do not call it bullying when it is done in a friendly or playful way.

Mothers were interviewed when children were 7,10 , and 12 years old and asked whether either twin had been bullied by another child, responding never, yes, or frequently. We combined mothers' reports at child age 7 and 10 to derive a measure of victimization during primary school. Mothers' reports when the children were 12 years old indexed victimization during secondary school. During private interviews with the children when they were 12 years old, the children indicated whether they had been bullied by another child during primary or secondary school. When a mother or a child reported victimization, the interviewer asked her to describe what happened. Notes taken by the interviewers were later checked by an independent rater to verify that the events reported could be classified as instances of bullying operationally defined as evidence of (a) repeated harmful actions, (b) between children, and (c) where there is a power differential between the bully and the victim (Shakoor et al., 2011). Although interrater reliability between mothers and children was only modest ( $\kappa=0.20-0.29)$, reports of victimization from both informants were similarly associated with children's emotional and behavioral problems, suggesting that each informant provides a unique but meaningful perspective on bullying involvement (Shakoor et al., 2011). We thus combined mother and child reports of victimization to capture all instances of bullying victimization for primary and secondary school separately: reported as not victimized by both mother and child; reported by either mother or child as being occasionally victimized; and reported as being occasionally victimized by both informants or as frequently victimized by either mother or child or both (Bowes et al., 2013). We then combined these primary and secondary school ratings to create a bullying victimization variable for the entire childhood period (5-12 years). Children who were never bullied in primary or secondary school or occasionally bullied during one of these time periods were coded as $0(55.5 \%)$; children who were occasionally bullied during primary and secondary school, or frequently bullied during one of these time periods were coded as 1 (35.6\%); and children who were frequently bullied at both primary and secondary school were coded as $2(8.9 \%)$.

Physical harm by an adult. When the twins were aged 5, 7, 10 , and 12, their mothers were interviewed about each twin's experience of intentional harm by an adult. At age 5 we used the standardized clinical protocol from the MultiSite Child Development Project (Dodge, Bates, \& Pettit, 1990; Lansford et al., 2002). At ages 7, 10, and 12 this interview was modified to expand its coverage of contexts for child harm. Interviews were designed to enhance mothers' comfort with reporting valid child maltreatment information, while also meeting researchers' responsibilities for referral under the UK Children Act. Specifically, mothers were asked whether either of their twins had been intentionally harmed (physically or sexually) by an adult or had contact with welfare agencies. If caregivers endorsed a question, interviewers made extensive notes on what had happened, and indicated whether physical and/or psychological harm had occurred. Under the UK Children Act, our responsibility was to secure intervention if maltreatment was current and ongoing. Such intervention on behalf of E-Risk families was carried out with parental cooperation in all but one case. No families left the study following intervention.

Over the years of data collection, the study developed a cumulative profile for each child, comprising the caregiver reports, recorded debriefings with interviewers who had coded any indication of maltreatment at any of the successive home visits, recorded narratives of the successive caregiver interviews, and information from clinicians whenever the study team made a child-protection referral. The profiles were reviewed at the end of the age 12 phase by two clinical psychologists. Initial interrater agreement between the coders exceeded $90 \%$, and discrepantly coded cases were resolved by consensus review. These were coded as follows: $0=$ no maltreatment at any age; $1=$ probable maltreatment at any age; and $2=$ definite maltreatment at any age. There were $15.4 \%$ of the children coded as probably being exposed to physical harm and $5.7 \%$ as definitely physically harmed by 12 years of age.

Physical neglect. The cumulative observations of the physical state of the home environment documented by the interviewers during home visits to the twins at ages 5, 7, 10, and 12 were reviewed by two raters for evidence of physical neglect. This was defined as any sign that the caretaker was not providing a safe, sanitary, or healthy environment for the child. This included the child not having proper clothing or food, as well as grossly unsanitary home environments. (However, 
this did not include a family living in a crime-ridden neighborhood for economic reasons.) Initial interrater agreement between the coders exceeded $85 \%$, and discrepantly coded cases were resolved by consensus review. Children with no evidence of physical neglect were coded as $0(90.9 \%)$, those for whom there was an indication of minor physical neglect were coded as $1(7.1 \%)$, and where there was evidence of severe physical neglect the children were coded as $2(2.0 \%)$.

\section{Results}

\section{Prevalence of victimization in adolescence}

Table 1 presents the rates of victimization between ages 12 and 18 years, as reported by the E-Risk study participants at age 18. Over half of the participants reported exposure to crime victimization $(51.7 \%)$ and to some form of peer/sibling victimization $(58.7 \%)$. However, only $15.4 \%(N=318)$ reported that their cotwin had been one of the perpetrators. Approximately one in five participants reported exposure from age 12 to 18 to Internet/mobile phone victimization $(20.6 \%)$ and to family violence $(19.3 \%)$ and slightly less reported sexual victimization $(16.4 \%)$ or maltreatment (14.8\%). A smaller percentage of participants reported neglect (6.4\%).

For comparison purposes, Table 1 also presents the lifetime rates of victimization for 11- to 17- year-olds across the United Kingdom collected by the NSPCC in 2009 (Radford et al., 2011). Although the two studies differed in important ways (e.g., we conducted face-to-face interviews whereas the

Table 1. Prevalence of adolescent victimization experiences among E-Risk participants and respondents in the NSPCC survey

\begin{tabular}{|c|c|c|}
\hline & $\begin{array}{c}\text { E-Risk } \\
\text { Sample }^{b} \\
(N=2066)\end{array}$ & $\begin{array}{c}\text { NSPCC National } \\
\text { Sample } \\
(N=2275)\end{array}$ \\
\hline Type of Victimization ${ }^{a}$ & $n(\%)$ & $n(\%)^{d}$ \\
\hline Crime & $1067(51.7)$ & $1437(62.2)$ \\
\hline Peer/sibling & $1212(58.7)$ & $1471(64.9)$ \\
\hline Internet/mobile phone & $425(20.6)$ & $319(13.3)$ \\
\hline Sexual & $338(16.4)$ & $285(16.5)$ \\
\hline Family violence & $398(19.3)$ & $342(19.8)$ \\
\hline Maltreatment & $306(14.8)$ & $358(20.7)$ \\
\hline Neglect & $132(6.4)$ & $229(13.3)$ \\
\hline
\end{tabular}

Note: E-Risk, Environmental Risk Longitudinal Twin Study; NSPCC, National Society for the Prevention of Cruelty to Children.

${ }^{a}$ We report whether any victimization experience was reported, within each type of victimization.

${ }^{b}$ Victimization between ages 12 and 18 reported by 18 -year-olds in the E-Risk Study.

${ }^{c}$ Lifetime victimization reported by 11 - to 17 -year-olds from across the United Kingdom, taken from Radford et al. (2011).

${ }^{d}$ These percentages are weighted back to the UK population to compensate for unequal sampling probabilities, and unequal responses by age group, gender, housing tenure, working status, region, and ethnic group (see Radford et al., 2011, for full details).
NSPCC survey used a computerized self-report version of the JVQ; we inquired about victimization over the past 6 years whereas the NSPCC survey provides lifetime rates; and our study participants are 18 years old whereas the NSPCC survey involved 11- to 17-year-olds), the prevalence rates of victimization are broadly comparable. The exceptions to this were maltreatment and neglect, which were reported less often by our participants. This is likely to be due to our prevalence rates being limited to adolescence, while the NSPCC survey captured both childhood and adolescent exposure, which could account for the higher rates in that survey.

Table 1 documents high rates of victimization according to the participants' reports, but these experiences are difficult to interpret without any discrimination as to whether they were mild or severe. Moving beyond the survey responses in Table 1, Table 2 presents information about the severity of victimization experiences as derived from evaluation and coding of the victimization dossiers compiled for our study participants. All findings presented in the remainder of this article refer to the severity-coded victimization experiences.

Approximately a quarter $(24.3 \%)$ of adolescents were exposed to severe levels of violence ("any physical violence") between ages 12 and 18 years; that is, to incidents directed toward themselves or that they witnessed involving other people that were likely to result in physical injuries or were life threatening. Turning to specific types of severe victimization experiences, just under one fifth reported exposure to severe forms of crime victimization $(19.3 \%)$ and $15.6 \%$ reported being frequently victimized by a peer or sibling. Exposure to severe and/or frequent family violence was reported by $12.1 \%$ of the participants, and Internet/mobile phone victimization by $6.5 \%$ of the adolescents. Severe maltreatment $(3.3 \%)$, contact sexual abuse $(2.6 \%)$, and extreme neglect $(2.2 \%)$ were the least common types of victimization in this sample during adolescence. The rates of exposure to victimization were almost identical among MZ and DZ twin participants in our cohort (see supplementary materials Part III).

Table 2 also presents the prevalence of each victimization type by gender and the association between gender and exposure to severity of victimization during adolescence. When calculating these associations using ordinal logistic regression, we accounted for the nonindependence of the twin observations using the Huber-White variance estimator (Williams, 2000). This adjusts the estimated standard errors in each test to account for the dependence in the data. Overall, males were exposed to more severe levels of physical violence than were females (see Table 2) and there were sex differences in the other types of victimization experienced by adolescents. Whereas exposure to crime and peer/sibling victimization was more common among males, exposure to Internet/mobile phone victimization, sexual victimization, and maltreatment was more common among females. Adolescent males and females did not differ in their exposure to family violence or neglect.

Participants from low-SES backgrounds were more like to experience severe exposure to nearly all types of victimiza- 
Table 2. Distribution of the severity of adolescent victimization experiences among males and females in the E-Risk study

\begin{tabular}{|c|c|c|c|c|c|}
\hline \multirow{3}{*}{$\begin{array}{c}\text { Type of } \\
\text { Victimization }\end{array}$} & \multicolumn{3}{|c|}{ Severity Rating } & \multirow[b]{2}{*}{ Males Vs. Females } & \multirow[b]{3}{*}{$p$} \\
\hline & Never & Some/Occasional & Severe/Frequent & & \\
\hline & $n(\%)$ & $n(\%)$ & $n(\%)$ & OR $(95 \% \mathrm{CI})$ & \\
\hline \multicolumn{6}{|c|}{ Any physical violence } \\
\hline All & $984(47.6)$ & $580(28.1)$ & $502(24.3)$ & \multirow{3}{*}{$0.53(0.44-0.65)$} & \multirow{3}{*}{$<.001$} \\
\hline Boys & 379 (38.6) & $316(32.2)$ & $286(29.2)$ & & \\
\hline Girls & $605(55.8)$ & $264(24.3)$ & $216(19.9)$ & & \\
\hline \multicolumn{6}{|l|}{ Crime } \\
\hline All & $998(48.3)$ & $670(32.4)$ & $398(19.3)$ & \multirow{3}{*}{$0.50(0.41-0.60)$} & \multirow{3}{*}{$<.001$} \\
\hline Boys & $378(38.5)$ & $369(37.6)$ & $234(23.9)$ & & \\
\hline Girls & $620(57.1)$ & $301(27.7)$ & $164(15.1)$ & & \\
\hline \multicolumn{6}{|l|}{ Peer/sibling } \\
\hline All & $872(42.2)$ & $870(42.1)$ & $323(15.6)$ & \multirow{3}{*}{$0.82(0.68-0.99)$} & \multirow{3}{*}{.040} \\
\hline Boys & $365(37.2)$ & $484(49.4)$ & $131(13.4)$ & & \\
\hline Girls & $507(46.7)$ & $386(35.6)$ & $192(17.7)$ & & \\
\hline \multicolumn{6}{|c|}{ Internet/mobile phone } \\
\hline All & 1644 (79.7) & $286(13.9)$ & $133(6.5)$ & \multirow{3}{*}{$1.79(1.41-2.27)$} & \multirow{3}{*}{$<.001$} \\
\hline Boys & $825(84.2)$ & $125(12.8)$ & $30(3.1)$ & & \\
\hline Girls & $819(75.6)$ & $161(14.9)$ & $103(9.5)$ & & \\
\hline \multicolumn{6}{|l|}{ Sexual } \\
\hline All & $1808(87.8)$ & $198(9.6)$ & $53(2.6)$ & \multirow{3}{*}{$1.94(1.44-2.62)$} & \multirow{3}{*}{$<.001$} \\
\hline Boys & $893(91.3)$ & $76(7.8)$ & $9(0.9)$ & & \\
\hline Girls & 915 (84.6) & $122(11.3)$ & $44(4.1)$ & & \\
\hline \multicolumn{6}{|l|}{ Family violence } \\
\hline All & $1676(81.3)$ & $136(6.6)$ & $250(12.1)$ & \multirow{3}{*}{$1.02(0.79-1.32)$} & \multirow{3}{*}{.851} \\
\hline Boys & $798(81.5)$ & $62(6.3)$ & $119(12.2)$ & & \\
\hline Girls & $878(81.1)$ & $74(6.8)$ & $131(12.1)$ & & \\
\hline \multicolumn{6}{|l|}{ Maltreatment } \\
\hline All & $1783(86.4)$ & $213(10.3)$ & $67(3.3)$ & \multirow{3}{*}{$1.50(1.13-2.00)$} & \multirow{3}{*}{.006} \\
\hline Boys & $872(89.0)$ & $76(7.8)$ & $32(3.3)$ & & \\
\hline Girls & $911(84.1)$ & 137 (12.7) & $35(3.2)$ & & \\
\hline \multicolumn{6}{|l|}{ Neglect } \\
\hline All & 1936 (93.9) & $80(3.9)$ & $46(2.2)$ & \multirow{3}{*}{$1.31(0.86-1.99)$} & \multirow{3}{*}{.208} \\
\hline Boys & 927 (94.7) & $34(3.5)$ & $18(1.8)$ & & \\
\hline Girls & $1009(93.2)$ & $46(4.3)$ & $28(2.6)$ & & \\
\hline
\end{tabular}

Note: OR, Proportional odds ratios derived from ordinal logistic regression adjusted for the nonindependence of twin observations; CI, confidence interval.

tion, including crime, family violence, maltreatment, and neglect (Table 3). However, they were only slightly more likely to be victimized by peers or siblings, and there was no social gradient in exposure to Internet/mobile phone or sexual victimization.

\section{Polyvictimization: Are adolescents likely to be exposed to multiple types of severe victimization?}

We tested the co-occurrence of different types of severe victimization during adolescence by estimating the odds of being exposed to one type of severe victimization given exposure to another type of severe victimization. The positive manifold in Table 4 reveals that participants who were exposed to one type of severe victimization in adolescence were much more likely to have been exposed to multiple other types of severe victimization. Four findings stand out. First, adolescents who experienced intrafamilial types of victimization were more likely to also experience victimization outside of the family home. Second, participants who were cyber/mobile-technology victims were also more likely to be exposed to victimization in the physical world. Third, the experience of neglect was not only strongly associated with physical maltreatment by adults but also linked to multiple other types of victimization. Fourth, these associations were independent of socioeconomic disparities in victimization exposure. That is, polyvictimization was not simply a function of concentrated exposure to violence among adolescents growing up in socioeconomically deprived circumstances.

To investigate the level of polyvictimization (i.e., of experiencing multiple types of severe victimization) during adolescence, we summed the number of different types of severe victimization experiences encountered by each participant. Around a third of participants experienced at least one of the seven types of severe victimization in adolescence (35.4\%). Of these, almost half (45.7\%) were exposed to multiple different types of severe victimization (Figure 1a). Female participants $(17.8 \%)$ were slightly more likely to have 
Table 3. Distribution of the severity of adolescent victimization experiences as a function of socioeconomic status (SES)

\begin{tabular}{|c|c|c|c|c|c|}
\hline \multirow{3}{*}{$\begin{array}{c}\text { Type of } \\
\text { Victimization }\end{array}$} & \multicolumn{3}{|c|}{ Severity Rating } & \multirow{3}{*}{$\frac{\text { Low Vs. Medium/High SES }}{O R(95 \% \mathrm{CI})}$} & \multirow[b]{3}{*}{$p$} \\
\hline & Never & Some/Occasional & Severe/Frequent & & \\
\hline & $n(\%)$ & $n(\%)$ & $n(\%)$ & & \\
\hline \multicolumn{6}{|c|}{ Any physical violence } \\
\hline Low & $274(39.7)$ & $187(27.1)$ & $230(33.3)$ & \multirow{2}{*}{$1.77(1.44-2.18)$} & \multirow{2}{*}{$<.001$} \\
\hline Medium/high & $710(51.6)$ & $393(28.6)$ & $272(19.8)$ & & \\
\hline \multicolumn{6}{|l|}{ Crime } \\
\hline Low & $290(42.0)$ & $220(31.8)$ & $181(26.2)$ & \multirow[t]{2}{*}{$1.59(1.30-1.96)$} & \multirow[t]{2}{*}{$<.001$} \\
\hline Medium/high & $708(51.5)$ & $450(32.7)$ & $217(15.8)$ & & \\
\hline \multicolumn{6}{|l|}{ Peer/sibling } \\
\hline Low & $266(38.6)$ & $299(43.3)$ & $125(18.1)$ & \multirow{2}{*}{$1.27(1.05-1.55)$} & \multirow[t]{2}{*}{.016} \\
\hline Medium/high & $606(44.1)$ & $571(41.5)$ & $198(14.4)$ & & \\
\hline \multicolumn{6}{|c|}{ Internet/mobile phone } \\
\hline Low & $539(78.1)$ & $99(14.4)$ & $52(7.5)$ & \multirow[t]{2}{*}{$1.17(0.91-1.49)$} & \multirow[t]{2}{*}{.224} \\
\hline Medium/high & $1105(80.5)$ & $187(13.6)$ & $81(5.9)$ & & \\
\hline \multicolumn{6}{|l|}{ Sexual } \\
\hline Low & $594(86.2)$ & $66(9.6)$ & $29(4.2)$ & \multirow[t]{2}{*}{$1.27(0.94-1.71)$} & \multirow[t]{2}{*}{.114} \\
\hline Medium/high & $1214(88.6)$ & $132(9.6)$ & $24(1.8)$ & & \\
\hline \multicolumn{6}{|l|}{ Family violence } \\
\hline Low & $535(77.5)$ & $47(6.8)$ & $108(15.7)$ & \multirow[t]{2}{*}{$1.46(1.12-1.89)$} & \multirow[t]{2}{*}{.005} \\
\hline Medium/high & $1141(83.2)$ & $89(6.5)$ & $142(10.4)$ & & \\
\hline \multicolumn{6}{|l|}{ Maltreatment } \\
\hline Low & $570(82.5)$ & $82(11.9)$ & $39(5.6)$ & \multirow[t]{2}{*}{$1.66(1.24-2.22)$} & \multirow[t]{2}{*}{.001} \\
\hline Medium/high & $1213(88.4)$ & $131(9.6)$ & $28(2.0)$ & & \\
\hline \multicolumn{6}{|l|}{ Neglect } \\
\hline Low & $624(90.4)$ & $37(5.4)$ & $29(4.2)$ & \multirow[t]{2}{*}{$2.34(1.53-3.56)$} & \multirow[t]{2}{*}{$<.001$} \\
\hline Medium/high & 1312 (95.6) & $43(3.1)$ & $17(1.2)$ & & \\
\hline
\end{tabular}

Note: OR, Proportional odds ratios derived from ordinal logistic regression adjusted for the nonindependence of twin observations; CI, confidence interval.

been classified as experiencing polyvictimization than were male participants (14.4\%; Figure 1b).

We further examined the phenomenon of polyvictimization by adopting a person-centered approach to adolescents' victimization experiences. Latent class analysis is a personcentered analytical approach that classifies individuals into groups or classes based on a profile of variables, in this case exposure versus no exposure to the seven types of severe

Table 4. The co-occurrence of different types of severe victimization experienced by adolescents

\begin{tabular}{|c|c|c|c|c|c|c|c|}
\hline $\begin{array}{l}\text { Type of Victimization } \\
\text { Experienced in } \\
\text { Adolescence }\end{array}$ & $\frac{\text { Crime }}{O R(95 \% \mathrm{CI})}$ & $\frac{\text { Peer/Sibling }}{\text { OR }(95 \% \mathrm{CI})}$ & $\begin{array}{c}\begin{array}{c}\text { Internet/Mobile } \\
\text { Phone }\end{array} \\
\text { OR }(95 \% \mathrm{CI})\end{array}$ & $\frac{\text { Sexual }}{O R(95 \% \mathrm{CI})}$ & $\begin{array}{c}\begin{array}{c}\text { Family } \\
\text { Violence }\end{array} \\
O R(95 \% \mathrm{CI})\end{array}$ & $\frac{\text { Maltreatment }}{\text { OR }(95 \% \mathrm{CI})}$ & $\frac{\text { Neglect }}{O R(95 \% \mathrm{CI})}$ \\
\hline Crime & - & $\begin{array}{c}3.84 \\
(3.36-4.38)\end{array}$ & $\begin{array}{c}3.50 \\
(2.99-4.09)\end{array}$ & $\begin{array}{c}3.35 \\
(2.76-4.07)\end{array}$ & $\begin{array}{c}2.29 \\
(1.96-2.67)\end{array}$ & $\begin{array}{c}3.47 \\
(2.88-4.18)\end{array}$ & $\begin{array}{c}2.87 \\
(2.18-3.76)\end{array}$ \\
\hline Peer/sibling & $\begin{array}{c}4.17 \\
(3.61-4.80)\end{array}$ & - & $\begin{array}{c}5.10 \\
(4.22-6.17)\end{array}$ & $\begin{array}{c}3.67 \\
(2.99-4.50)\end{array}$ & $\begin{array}{c}2.08 \\
(1.78-2.44)\end{array}$ & $\begin{array}{c}2.50 \\
(2.09-2.99)\end{array}$ & $\begin{array}{c}2.03 \\
(1.59-2.60)\end{array}$ \\
\hline Internet/mobile phone & $\begin{array}{c}3.53 \\
(3.01-4.14)\end{array}$ & $\begin{array}{c}5.04 \\
(4.16-6.11)\end{array}$ & - & $\begin{array}{c}3.31 \\
(2.74-4.01)\end{array}$ & $\begin{array}{c}1.70 \\
(1.43-2.02)\end{array}$ & $\begin{array}{c}2.32 \\
(1.94-2.77)\end{array}$ & $\begin{array}{c}1.76 \\
(1.38-2.25)\end{array}$ \\
\hline Sexual & $\begin{array}{c}4.20 \\
(3.40-5.20)\end{array}$ & $\begin{array}{c}4.37 \\
(3.46-5.51)\end{array}$ & $\begin{array}{c}3.86 \\
(3.06-4.86)\end{array}$ & - & $\begin{array}{c}2.34 \\
(1.85-2.97)\end{array}$ & $\begin{array}{c}3.88 \\
(2.99-5.04)\end{array}$ & $\begin{array}{c}3.23 \\
(2.39-4.38)\end{array}$ \\
\hline Family violence & $\begin{array}{c}2.03 \\
(1.78-2.31)\end{array}$ & $\begin{array}{c}1.79 \\
(1.59-2.03)\end{array}$ & $\begin{array}{c}1.59 \\
(1.38-1.84)\end{array}$ & $\begin{array}{c}1.94 \\
(1.64-2.30)\end{array}$ & - & $\begin{array}{c}3.40 \\
(2.90-3.99)\end{array}$ & $\begin{array}{c}3.52 \\
(2.82-4.40)\end{array}$ \\
\hline Maltreatment & $\begin{array}{c}4.35 \\
(3.51-5.41)\end{array}$ & $\begin{array}{c}2.45 \\
(2.03-2.97)\end{array}$ & $\begin{array}{c}2.48 \\
(2.04-3.03)\end{array}$ & $\begin{array}{c}3.74 \\
(2.91-4.80)\end{array}$ & $\begin{array}{c}5.14 \\
(4.02-6.58)\end{array}$ & - & $\begin{array}{c}7.44 \\
(5.63-9.85)\end{array}$ \\
\hline Neglect & $\begin{array}{c}3.20 \\
(2.40-4.25)\end{array}$ & $\begin{array}{c}1.98 \\
(1.58-2.47)\end{array}$ & $\begin{array}{c}1.93 \\
(1.54-2.42)\end{array}$ & $\begin{array}{c}2.87 \\
(2.10-3.93)\end{array}$ & $\begin{array}{c}4.84 \\
(3.53-6.64)\end{array}$ & $\begin{array}{c}7.61 \\
(5.66-10.24)\end{array}$ & - \\
\hline
\end{tabular}

Note: The victimization types listed in the first column are the predictor variables. OR, Proportional odds ratios derived from ordinal logistic regressions are adjusted for sex, socioeconomic status, and the nonindependence of twin observations; CI, confidence interval. 
(a) Full Sample

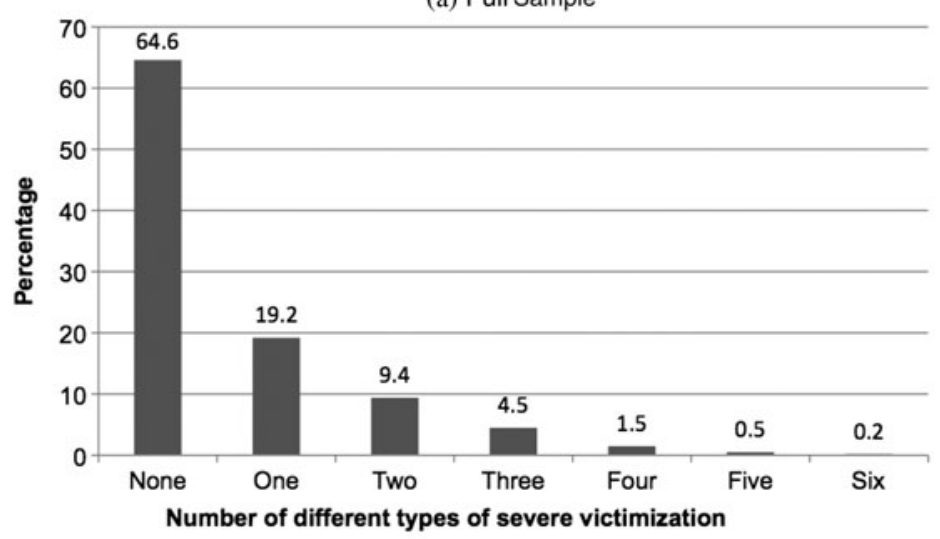

(b) By Gender

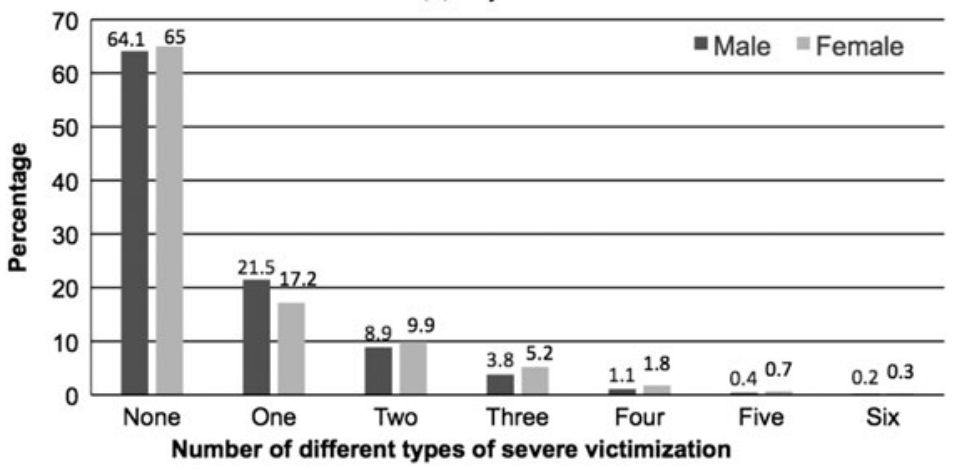

Figure 1. Number of different types of severe victimization experienced by adolescents in (a) the full sample $(N=2,059)$ and (b) among males $(N=978)$ and females $(N=1,081)$.

victimization. To ensure replicability of results, we randomly selected one twin from each family to include in initial analyses; the remaining twin was reserved for replication. In addition, because we were interested in the profiles of individuals who were victimized, we excluded participants who did not experience any severe forms of victimization. These selection criteria resulted in two subsamples of $N=365$ and $N=365$ individuals. Latent class analysis was conducted in MPlus v7.3. In each subsample, we examined fit statistics for two to six groups (Table 5). In the first subsample, the four-class solution was the preferred solution; in the second subsample, both the four- and five-class solutions were acceptable. Upon further examination of the profiles in the second subsample, we opted to retain the more parsimonious solution. Final membership classification was determined using the complete sample of $N=730$ individuals in a four-class model that simultaneously accounted for twin clustering. The model fit well: entropy $=0.85$; Lo-Mendell-Rubin adjusted likelihood ratio test for three versus four classes $=113.85, p<$ .001; Lo-Mendell-Rubin adjusted likelihood ratio test for four versus five classes $=38.70, p<.001$.

The four groups were defined by unique victimization profiles (Table 6). The first group was defined by exposure to crime; $84 \%$ of individuals classified into this group experienced exposure only to crime victimization, and the remaining $16 \%$ experienced one other type of victimization (mal- treatment, sexual victimization, or Internet/mobile phone victimization). The second group was defined by exposure to family violence; $59 \%$ of individuals classified into this group experienced exposure only to family violence; $22 \%$ experienced family violence and crime victimization; and $19 \%$ experienced family violence and other forms of victimization. The remaining groups were defined by profiles of polyvictimization. Specifically, the third group was defined by peer/sibling victimization in which the vast majority of adolescents also experienced at least one other form of victimization, and the fourth group of adolescents experienced multiple varieties of victimization.

\section{Revictimization: Are victimized children likely to be revictimized in adolescence?}

Cohort members who were exposed to domestic violence, bullied by peers, physically harmed by an adult, or neglected as a child were significantly more likely (a) to be exposed to severe violence in adolescence, (b) to experience each of the different types of severe victimization in adolescence, and (c) to experience polyvictimization (i.e., they were exposed to a greater variety of different types of severe victimization; Table 7). Victimization in early life did not simply show homotypic continuity, a term that we apply here to refer to the continuity of similar experiences. Rather, every type of se- 
Table 5. Fit statistics for the latent class analysis in two random subsamples of victimized adolescents in the Environmental Risk Longitudinal Twin Study

\begin{tabular}{|c|c|c|c|c|c|c|c|c|c|}
\hline \multirow[b]{2}{*}{ No. of Classes } & \multirow[b]{2}{*}{ Log Likelihood } & \multirow[b]{2}{*}{ AIC } & \multirow[b]{2}{*}{$\mathrm{BIC}$} & \multicolumn{3}{|c|}{ Likelihood Ratio $\chi^{2}$} & \multirow[b]{2}{*}{ Entropy } & \multicolumn{2}{|c|}{ LMR Adj. LRT } \\
\hline & & & & Estimate & $d f$ & $p$ & & Estimate & $p$ \\
\hline \multicolumn{10}{|c|}{ Subsample $1(N=365)$} \\
\hline 2 & -1170.517 & 2371.003 & 2429.532 & 256.982 & 112 & $<.001$ & 1.000 & 69.853 & $<.001$ \\
\hline 3 & -1131.452 & 2308.903 & 2398.601 & 178.852 & 104 & $<.001$ & 0.757 & 76.509 & .034 \\
\hline 4 & -1093.750 & 2249.500 & 2370.397 & 103.449 & 96 & .284 & 0.881 & 73.838 & $<.001$ \\
\hline 5 & -1081.108 & 2240.984 & 2392.312 & 77.164 & 88 & .764 & 0.947 & 24.760 & .540 \\
\hline 6 & -1070.501 & 2235.003 & 2418.298 & 56.951 & 80 & .976 & 0.946 & 20.773 & .237 \\
\hline \multicolumn{10}{|c|}{ Subsample $2(N=365)$} \\
\hline 2 & -1150.149 & 2330.298 & 2388.796 & 211.678 & 111 & $<.001$ & 0.758 & 91.751 & $<.001$ \\
\hline 3 & -1102.563 & 2251.126 & 2340.824 & 117.589 & 102 & .139 & 0.866 & 93.197 & $<.001$ \\
\hline 4 & -1077.613 & 2217.227 & 2338.123 & 87.700 & 96 & .715 & 0.857 & 48.864 & .007 \\
\hline 5 & -1067.970 & 2213.940 & 2366.036 & 68.413 & 88 & .940 & 0.775 & 18.886 & .037 \\
\hline 6 & -1060.565 & 2215.131 & 2398.426 & 53.604 & 80 & .990 & 0.927 & 14.502 & .216 \\
\hline
\end{tabular}

Note: AIC, Akaike information criterion; BIC, Baysian information criterion; LMR Adj. LRT, Lo-Mendell-Rubin adjusted likelihood ratio test.

vere victimization in childhood was broadly related to both the same and other, different, types of severe victimization throughout adolescence.

Turning to exposure to severe physical violence in adolescence, we observe remarkable continuity in the lives of children and adolescents, whether looking forward or looking backward. In a follow-forward longitudinal analysis, Figure 2a shows that $36.6 \%$ of children who were exposed to repeated episodes of domestic violence, $40.4 \%$ who were frequently bullied, $55.7 \%$ who were physically harmed, and $51.4 \%$ who were neglected, grew up to be exposed to severe physical violence in adolescence. In a follow-back longitudinal analysis, Figure $2 \mathrm{~b}$ shows that of adolescents who were exposed to severe physical violence, $25.9 \%$ had experienced repeated exposure to domestic violence during childhood, $14.8 \%$ were frequently bullied during childhood, $13.6 \%$ were physically harmed, and
$3.6 \%$ were neglected. Only a small proportion $(12.9 \%)$ of those exposed to severe physical violence in adolescence had not been bullied or experienced, even mild forms of, maltreatment, physical domestic violence, or neglect in childhood. Broadly speaking, victimization is not something that goes away with time and is not something that often comes out of nowhere; it is a stable experiential pattern in the lives of many young people.

\section{Genetic and environmental contributions to adolescent victimization}

Table 8 shows the polychoric within-pair correlations for MZ twins and DZ twins separately for their exposure to each type of victimization during adolescence. Comparing MZ and DZ correlations allows us to estimate the relative contributions of

Table 6. Percentage of adolescents experiencing each type of victimization in the four classes/groups of victimization

\begin{tabular}{lcccc}
\hline \hline Type of Victimization & $\begin{array}{c}\text { Crime-Related } \\
\text { Victimization } \\
(N=190)\end{array}$ & $\begin{array}{c}\text { Family Violence } \\
\text { Victimization } \\
(N=158)\end{array}$ & $\begin{array}{c}\text { Polyvictimization I } \\
(N=329)\end{array}$ & $\begin{array}{c}\text { Polyvictimization II } \\
(N=53)\end{array}$ \\
\hline Crime & $100 \%$ & $26 \%$ & $39 \%$ & $86 \%$ \\
Peer/sibling & $0 \%$ & $0 \%$ & $86 \%$ & $35 \%$ \\
Internet/mobile phone & $7 \%$ & $4 \%$ & $31 \%$ & $13 \%$ \\
Sexual & $2 \%$ & $1 \%$ & $9 \%$ & $27 \%$ \\
Family violence & $0 \%$ & $100 \%$ & $18 \%$ & $66 \%$ \\
Maltreatment & $3 \%$ & $6 \%$ & $1 \%$ & $76 \%$ \\
Neglect & $0 \%$ & $6 \%$ & $2 \%$ & $45 \%$ \\
\hline \hline
\end{tabular}




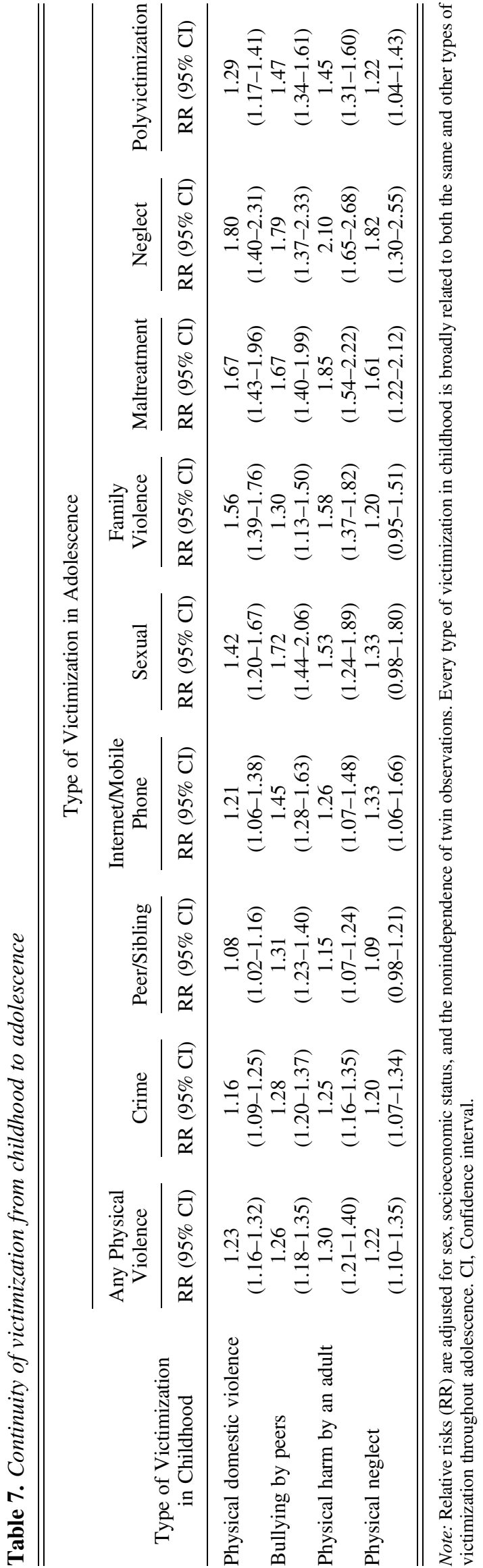

genetic and environmental factors to variation in participants' exposure to adolescent victimization. We examined the genetic and environmental influences on severity of victimization by decomposing variation in each form of victimization into that explained by additive genetic (A), shared environmental (C; environmental effects common to both twins), and nonshared environmental (E; environmental effects unique to each twin) factors. Victimization severity was treated as ordinal, and we used the threshold model for liabilities (Neale \& Cardon, 1992) to parameterize the model. In this case, the measured ordered categorical variables have no freely estimated residual variances and are represented as normally distributed latent response variables underlying the categorical outcome; the standard ACE variance and covariance restrictions are placed on these latent variables. ACE models were estimated in MPlus v7.3.

The parameter estimates varied by type of victimization (see Table 8). We observed the largest genetic influences on maltreatment and neglect, followed by peer/sibling victimization and crime victimization. Nonshared environmental influences were pronounced on all forms of victimization, suggesting that unique events and experiences were likely to put a given adolescent in harm's way; this was especially the case with regard to peer, cyber, and sexual victimization. In contrast to what is often reported in behavioral genetics, we detected consistent and substantial shared environmental influences on adolescents' victimization experiences, suggesting that growing up in certain families and communities does contribute to increased victimization risk.

\section{Discussion}

The aim of this report was to integrate best practices in survey research with optimal approaches to measuring human life stress, in the service of advancing the study of children's and adolescents' victimization experiences. Specifically, we combined a widely used self-report questionnaire (Finkelhor et al., 2011; Hamby et al., 2004) with an investigator-based rating system adapted from a comprehensive interview about stressful and traumatic childhood events (Bifulco, Brown, \& Harris, 1994). This combined method was successfully implemented with 2,066 18-year-olds who are participants in a nationally representative longitudinal twin cohort study. We demonstrated that our pragmatic approach of combining the brevity of a self-report questionnaire with the relative objectivity of a standardized investigator-based coding system can be successfully applied to characterize the severity of multiple types of adolescent victimization experiences with high levels of interrater reliability. By utilizing the optimal features of both assessment traditions, we are hopeful that this combined tool will bring both greater rigor and richness to the measurement of victimization in a manner that is scalable to large population-based studies.

Our approach is not without limitations. First, we relied on adolescents to tell us about their own victimization experiences, and such self-reports may be biased (Dohrenwend, 
(a) Follow-forward analysis: Percentage of victimized children who grew up to be exposed to severe physical violence in adolescence

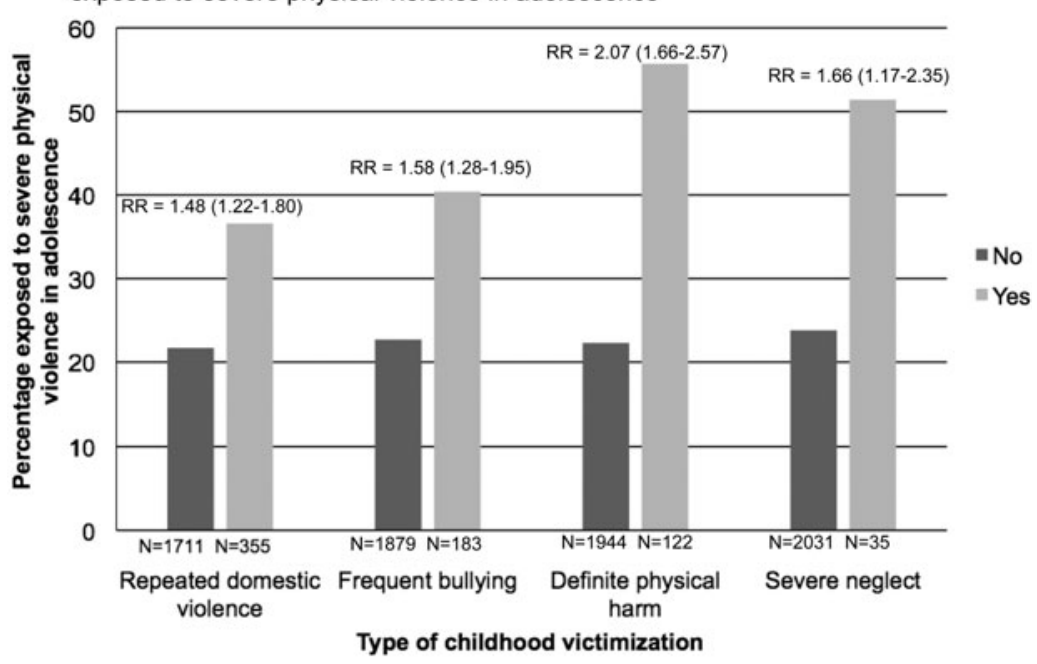

(b) Follow-back analysis: Percentage of adolescents exposed to severe physical violence who had also been victimized in childhood

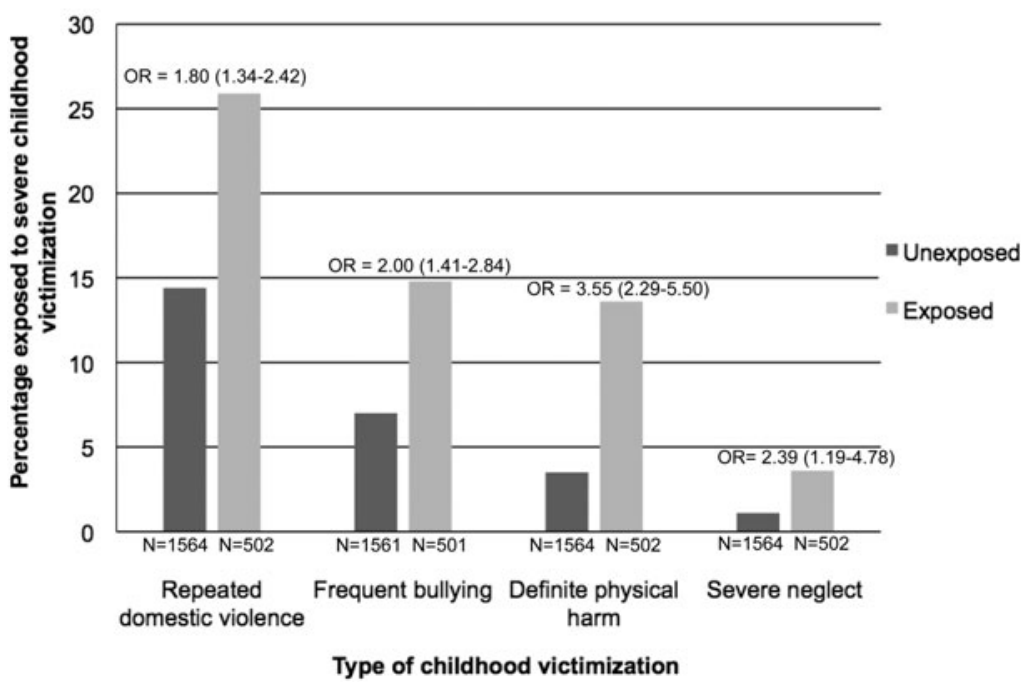

Figure 2. Violence exposure in adolescence (a) looking forward and (b) looking backward in the lives of victimized youth. Associations between childhood victimization and adolescent violence exposure are expressed as relative risks (RR) and between adolescent violence exposure and previous childhood victimization as odds ratios $(O R)$ with $95 \%$ confidence intervals adjusted for sex, socioeconomic status, and the nonindependence of twin observations.

2006; Grant et al., 2004; Hepp et al., 2006). However, we tried to minimize subjectivity by (a) conducting a face-toface interview, which provided the opportunity for the respondent and interviewer to clarify that the questions were understood as they were intended to be (Hardt \& Rutter, 2004; Schwarz, 2007), and (b) using an investigator-based standardized coding system to rate the severity of victimization experiences (Brown, 1974; Brown \& Harris, 1978). Second, given time constraints, our interview only queried details about each participant's "worst experience" within each victimization category. Third, it should be noted that even more fine-grained information could be elicited in the context of our combined method. For instance, more details about the victim's relationship with the perpetrator and the number of reoccurrences of the same victimization exposure may be important to evaluate in developmental psychopathology (Barnett et al., 1993; Fisher et al., 2010; Matta Oshima, Jonson-Reid, \& Seay, 2014; Thornberry, Ireland, \& Smith, 2001). Fourth, we used a face-to-face interview, and it is possible that some individuals may be less likely to disclose when questioned in person due to embarrassment (Della Femina, Yeager, \& Lewis, 1990). However, similar rates of abuse have been reported for self-report and interview-based versions of the same measure (Bifulco, Bernazzani, Moran, \& Jacobs, 2005). We welcome application of this assessment method in other studies to further test its feasibility, reliability and ease of use, further refine the anchor points (as advocated by Barnett et al., 1993), and extend its scope. 
Table 8. Within-pair polychoric correlations and genetic and environmental parameter estimates for univariate models of severity of adolescent victimization

\begin{tabular}{|c|c|c|c|c|c|c|c|c|}
\hline \multirow[b]{2}{*}{ Type of Victimization } & \multicolumn{2}{|c|}{$\begin{array}{l}\text { Cross-Twin } \\
\text { Correlation }\end{array}$} & \multicolumn{6}{|c|}{ Standardized Variance Components } \\
\hline & $r$ & $95 \% \mathrm{CI}$ & $\mathrm{A}^{2}$ & $95 \% \mathrm{CI}$ & $\mathrm{C}^{2}$ & $95 \% \mathrm{CI}$ & $E^{2}$ & $95 \% \mathrm{CI}$ \\
\hline Any physical violence & & & 0.22 & $0.00-0.46$ & 0.35 & $0.14-0.56$ & 0.43 & $0.35-0.51$ \\
\hline Monozygotic & .57 & $0.49-0.65$ & & & & & & \\
\hline $\begin{array}{l}\text { Dizygotic } \\
\text { Crime }\end{array}$ & .46 & $0.36-0.56$ & & & & & & \\
\hline $\begin{array}{l}\text { Crime } \\
\text { Monozygotic }\end{array}$ & .53 & $0.45-0.62$ & 0.30 & $0.04-0.56$ & 0.23 & $0.01-0.46$ & 0.47 & $0.39-0.55$ \\
\hline Dizygotic & .39 & $0.28-0.49$ & & & & & & \\
\hline Peer/sibling & & & 0.34 & $0.07-0.61$ & 0.12 & $0.00-0.35$ & 0.54 & $0.45-0.62$ \\
\hline Monozygotic & .47 & $0.38-0.55$ & & & & & & \\
\hline Dizygotic & .29 & $0.19-0.40$ & & & & & & \\
\hline Internet/mobile phone & & & 0.25 & $0.00-0.65$ & 0.17 & $0.00-0.51$ & 0.57 & $0.45-0.70$ \\
\hline Monozygotic & .42 & $0.30-0.55$ & & & & & & \\
\hline Dizygotic & .30 & $0.14-0.46$ & & & & & & \\
\hline Sexual & & & 0.28 & $0.00-0.77$ & 0.19 & $0.00-0.59$ & 0.53 & $0.37-0.69$ \\
\hline Monozygotic & .47 & $0.30-0.63$ & & & & & & \\
\hline Dizygotic & .33 & $0.14-0.51$ & & & & & & \\
\hline Family violence & & & 0.17 & $0.00-0.52$ & 0.42 & $0.12-0.72$ & 0.41 & $0.30-0.52$ \\
\hline Monozygotic & .59 & $0.47-0.70$ & & & & & & \\
\hline Dizygotic & .50 & $0.36-0.64$ & & & & & & \\
\hline Maltreatment & & & 0.71 & $0.61-0.80$ & - & - & 0.30 & $0.20-0.39$ \\
\hline Monozygotic & .72 & $0.62-0.82$ & & & & & & \\
\hline Dizygotic & .25 & $0.05-0.45$ & & & & & & \\
\hline Neglect & & & 0.47 & $0.00-0.96$ & 0.29 & $0.00-0.73$ & 0.25 & $0.13-0.37$ \\
\hline Monozygotic & .76 & $0.63-0.88$ & & & & & & \\
\hline Dizygotic & .52 & $0.30-0.75$ & & & & & & \\
\hline
\end{tabular}

Note: $r$, Polychoric correlation; CI, confidence interval; A, additive genetic; $\mathrm{C}$, shared environment; E, nonshared environment.

\section{Prevalence of adolescent victimization}

Traditionally each type of victimization measured by the JVQ is considered to have been experienced if a participant endorses at least one item in the relevant section (Finkelhor et al., 2011; Hamby et al., 2004). Using this scoring method, we found that the estimated prevalence rates of victimization exposure between 12 and 18 years in our cohort of 18-year-olds were broadly comparable to rates reported by the UK NSPCC in a cross-sectional survey of 11- to 17-year-olds (Radford et al., 2011). We found slightly lower rates of maltreatment and neglect in our sample, which likely reflects the fact that the NSPCC survey inquired about victimization experiences from birth onward, whereas we only inquired about experiences occurring between ages 12 and 18 years. We found higher rates of Internet/mobile phone victimization in our sample, which could be accounted for by the fact that this type of victimization occurs more often in adolescence than in childhood and that it may be on the rise (our data collection period occurred a few years after the NSPCC survey was conducted; Jones, Mitchell, \& Finkelhor, 2012). Despite discrepancies in study design, the reported rates of the different types of victimization in E-Risk and in the UK NSPCC survey are reassuringly similar.
As others have commented (Gilbert et al., 2009), a concerning proportion of children and adolescents are victimized. According to the survey administered to our research participants, close to $73 \%$ of adolescents have been exposed to some form of victimization. According to our rating system of severity, approximately one in three adolescents were classified as having experienced at least one type of severe victimization between ages 12 and 18 years (crime victimization, peer/sibling victimization, Internet/mobile phone victimization, sexual victimization, family violence, maltreatment, or neglect). These results imply that victimization surveys capture both severe as well as more minor experiences and that there is considerable heterogeneity in the victimization experiences captured in surveys of youth. This discrepancy does not imply that one estimate is better or worse. Both the survey and the clinical interview parent measures that we drew upon were themselves designed to be fit for their original purpose, and had documented reliability and validity. Our offspring measure showed "hybrid vigor," blending the best advantages of both established approaches to documenting and studying victimization. The higher survey count is required for estimating the size of the problem for prevention planning. At the prevention stage, before an incident occurs, it is not possible to know how serious the incident will become, and all forms of victim- 
ization should be prevented. After an incident occurs, and when researchers seek to carry out research to inform postvictimization treatment, it is necessary to discriminate the severity of the victimization. Such refinement to exposure measurement is fundamental to a better understanding of the sequelae of stress and to studies that seek to reliably identify and replicate resiliency factors, whether in the genome, in supportive relationships, or in the wider community (Barnett et al., 1993; Monroe \& Reid, 2008; Wertz \& Pariante, 2014).

\section{Revictimization from childhood through adolescence}

A striking finding from our longitudinal analysis is that victims of childhood maltreatment are victimized again in adolescence. This phenomenon of revictimization has been documented before (e.g., Widom, Czaja, \& Dutton, 2008; Radford et al., 2013), but much of the evidence about revictimization comes from cross-sectional studies that collect victims' retrospective reports about their childhood experiences or from studies that rely on cases identified by child protection agencies. Moreover, many studies tend to focus narrowly on victims of specific experiences (e.g., sexual abuse or assault). Using prospective data and guarding against singlesource reporting biases, here we show that victimization experiences in childhood, as reported by primary caregivers, are strongly predictive of self-reports of experiences of victimization occurring in adolescence. Two findings stand out. First, children who suffer one type of victimization are likely to experience diverse forms of victimization at later points in adolescence. Second, adolescents who are exposed to severe physical violence are likely to have experienced diverse forms of victimization at earlier points in development. These results point to three conclusions: (a) victimization shows considerable continuity in the lives of children and adolescents; (b) it does not remain true to type during the first two decades of life; and (c) there does not appear to be a specific "gateway" via certain types of childhood victimization to revictimization (Finkelhor, Ormrod, \& Turner, 2007).

In terms of theory, the phenomenon of revictimization demands more vigorous attention, not only so that we may understand how it comes about, but also so that we may be better able to predict and prevent it. Multiple explanations have been invoked to account for the phenomenon of revictimization, ranging from ecological systems theory (e.g., environmental factors that create victimization vulnerability are stable over victims' lives) to psychological theories (e.g., learned helplessness), but few studies directly test multiple and competing explanations.

In terms of research design, evidence of revictimization calls attention to the need to reliably assess victimization experiences across multiple developmental periods. For example, much research attention is currently focused on biological pathways linking early life stress to later health (Moffitt \& the Klaus Grawe ThinkTank, 2013). However, if revictimization is as ubiquitous as our data show, it suggests that theorizing about sensitive periods needs to be balanced by the pos- sibility that many of the effects attributable to the biological embedding of early life stress may actually operate by increasing exposure to additional forms of victimization and creating a greater cumulative stress load.

In terms of preventing revictimization, our findings suggest that efforts should be focused on children physically abused by their caregivers. In our sample, over half of those physically maltreated in childhood were exposed to severe physical violence in adolescence. This is consistent with previous studies that have suggested maltreated children are particularly vulnerable to being victimized again and to experience other forms of victimization including physical assault (Radford et al., 2013; Widom et al., 2008). Targeting interventions at children identified as having been maltreated by an adult may thus reduce revictimization rates in adolescence, but this hypothesis requires testing.

\section{Polyvictimization among adolescents}

Victimologists have noted that polyvictimization is a neglected component of children's victimization experiences, and our findings amplify the call to broaden the focus in developmental psychopathology to the wider range of experiences in which victimization events occur (Finkelhor et al., 2007; Finkelhor, Turner, Ormrod, \& Hamby, 2009). Evidence of polyvictimization complicates research that seeks to specify the effects of victimization and to develop intervention and treatment programs. Most research that traces the consequences of victimization, and most theoretical explanations of such putative effects, are organized around a single type of victimization (e.g., bullying, physical abuse, sexual victimization, or neglect). However, the majority of severe victimization experiences in our cohort occurred to youth who experienced at least one other type of victimization. Whereas some youth were only victimized by crime and other youth were only exposed to violence between family members, only a minority of youth solely experienced victimization by peers, and practically no youth experienced instances of maltreatment, sexual victimization, or neglect that were not embedded in the context of other victimization experiences.

We see parallels between the challenge posed by polyvictimization and the now-familiar challenge posed by comorbidity. A persistent challenge in mental health research and treatment is the coexistence of two or more conditions or disorders; in community samples, almost half of individuals who meet diagnostic criteria for one disorder meet diagnostic criteria for another disorder, and the rates are higher in clinical samples (Clark, Watson, \& Reynolds, 1995; Newman et al., 1996). Among the implications of such high rates of comorbidity is that (a) studying "pure" cases may offer an unrepresentative picture of the disorder; (b) it is very difficult to identify disorder-specific causes, correlates, and sequelae when disorders co-occur at such high rates; and (c) transdiagnostic approaches to mental health deserve more attention (Caspi et al., 2014). In parallel, we suggest three implications for victimization research. First, most "pure" instances of victimiza- 
tion may not represent the developmental landscape or experiential history of victimization. Second, it will be challenging for researchers to identify correlates and sequelae that are unique to particular victimization experiences. When claims of specificity are sought, empirical verification may need to include not only unexposed controls but also controls that have been exposed to other forms of victimization. Third, treatment options must attend to the ubiquitous experience of polyvictimization, on top of pervasive revictimization. It may be that the most expedient approach to interrogating the psychological and physical consequences of victimization and to developing treatment options is to attend to the cumulative experience of such exposures.

\section{Genetic and environmental influences on victimization exposure}

Traditional research treats victimization as an environmental exposure that threatens healthy development. However, the unidirectional environment $\rightarrow$ person connection has been challenged by evidence that many putative environmental exposures are, to some extent, under genetic control (Plomin \& Bergeman, 1991). Specifically, genetically informative designs (twin and adoption studies) that have been used to decompose variation in measures of the environment suggest that differences between individuals in their child-rearing experiences, in their relationships with peers, and even in their risk of being exposed to stressful life events are partially heritable (Kendler \& Baker, 2007; Plomin, 1994). Models in victimology (e.g., lifestyle-exposure theory) recognize that victimization is not evenly distributed in the population and that there exist both high-risk places and high-risk persons. Initial research into the genetic origins of adolescent victimization showed that genetic factors may account for up to 50\% of the variation in criminal victimization (e.g., being threatened with a knife; Beaver, Boutwell, Barnes, \& Cooper, 2009; Beaver, Boutwell, Barnes, DeLisi, \& Vaughn, 2013), but victimization was difficult to disentangle from perpetration. Although some believe that seeking to identify the causes of victimization is tantamount to blaming the victim, we think that our twin study provides a unique vantage point for exploring the causes of victimization by systematically surveying multiple types of victimization. The results pointed to a highly nuanced view of adolescent victimization, with meaningful differences in the origins of different types of experiences and with implications for understanding genetic and environmental influences on adolescent development.

Turning to genetic effects, we found that peer/sibling and crime victimization showed significant, but modest heritabilities. Maltreatment was under stronger genetic influence, suggesting that heritable characteristics of the victims influence their likelihood of being physically maltreated in adolescence, because these characteristics either evoke maltreatment from adults or lead victims to end up in risky situations. This is intriguing because a previous report from our group found that maltreatment up to 12 years of age in the current sample was largely influenced by environmental factors shared between the twins, with almost no effect of genetic factors (Jaffee, Caspi, Moffitt, \& Taylor, 2004). It is difficult to know whether this difference reflects a developmental shift or a methodological difference. On the one hand, it is conceivable that these forms of victimization during adolescence are more often an adults' response to adolescent instigation than are such experiences in childhood. On the other hand, our analysis of maltreatment up to age 12 years relied on interviews conducted with the twins' mothers, whereas our analysis of maltreatment in adolescence relied on interviews with the twins themselves. This could potentially have led to the different results we found regarding genetic influence on maltreatment in these two developmental periods. We are not able to disentangle these alternative interpretations. Finally, and in marked contrast to other forms of victimization, sexual victimization in adolescence was not under significant genetic influence and was mostly explained by unique environmental risk factors, specific to each twin in a pair.

Turning to the environment, our findings contribute to a nascent reassessment of socialization research pointing to the importance of shared environmental influences on adolescent development (Burt, 2009). A long-standing controversy set in motion by behavioral geneticists is the claim that most of the similarity between siblings is due to genetic effects and that most of the differences between siblings are due to environmental effects; that is, shared environmental experiences do not create similarities between siblings. At its extreme, this evidence has been used to challenge the notion that families and communities are the most important factors in children's development (Harris, 1998). Our data suggest otherwise, and show that family-wide shared risk factors accounted for approximately $10 \%-40 \%$ of the variance in adolescents' victimization experiences. One possible reason for our evidence that shared environmental factors give rise to similar victimization experiences may have to do with our measurement approach. Most studies that have examined genetic influences on environmental experiences have relied on self-reports of the environment. Estimates of genetic influence on measures of the environment are much smaller in studies that have measured the environment via more direct means (e.g., using observational measures). This has led to the suggestion that the so-called nature of nurture may to some extent reflect individuals' heritable propensity to perceive the environment in particular ways (Plomin, 1994). In our research, we have taken a middle course, using an investigator-based system to determine and interpret the environmental experience. A second possibility relates to the fact that we measured severe victimization experiences. Whereas mild exposures may be less influenced by variation in the "average expectable" environment, grossly substandard or dysfunctional environments may shape extreme experiences (Scarr, 1993). Finally, and relatedly, whereas historically most twin studies have not deliberately or adequately sampled the extreme ranges of adversity, we sought to ensure that the full range of contemporary Western children's environments was adequately sampled. Restriction of environmental range may 
have led previous studies to underestimate the importance of shared environmental experiences.

The findings about genetic and environmental effects on victimization have two implications. First, it appears that much of the variation in victimization experiences is the result of being exposed to risky environments, and not the product of victims' heritable characteristics. Identifying these environmental risk factors should be a priority in order to hasten preventative interventions. Second, many adolescents growing up in the same households and neighborhoods are differentially exposed to victimization. Such discordance offers an important opportunity for basic science. It is clear that etiologic studies that seek to understand environmental effects on mental and physical health need to account for genetic influences on environmental experiences. Studies of differentially exposed twins provide unique purchase on unconfounded genetic and environmental effects on health (van Dongen, Slagboom, Draisma, Martin, \& Boomsma, 2013), especially in relation to better understanding the consequences of violence victimization.

Many young people who are mistreated by an adult, victimized by bullies, criminally assaulted, or who witness domestic violence react by developing behavioral, emotional, or learning problems. Increasingly, it is emerging that such adverse experiences can lead to hidden physical alterations inside a child's body, alterations that may have adverse effects on life-long health. This evidence has encouraged stress-biology research and intervention science to join forces to tackle the problem of victimization (Moffitt \& the Klaus Grawe ThinkTank, 2013). Both basic and translational science will be advanced by flexible and rich measurement tools that can be widely applied in different settings. Here we have documented the feasibility of combining a widely used self-report questionnaire with an investigator-based rating system to characterize victimization experiences. Too often research on important problems is stymied by measurement impasses created by disciplinary preferences (survey researchers vs. clinical scientists) and imposed by practical considerations (e.g., insufficient time). We hope that the combined approach that we have introduced, borrowing on two valuable traditions for measuring victimization, will offer converging opportunities for scientists and practitioners of all stripes to coordinate their efforts.

\section{Supplementary Material}

To view the supplementary material for this article, please visit http://dx.doi.org/S0954579415000838

\section{References}

Arseneault, L., Walsh, E., Trzesniewski, K., Newcombe, R., Caspi, A., \& Moffitt, T. E. (2006). Bullying victimization uniquely contributes to adjustment problems in young children: A nationally representative cohort study. Pediatrics, 118, 130-138.

Barnett, D., Manly, J. T., \& Cicchetti, D. (1993). Defining child maltreatment: The interface between policy and research. In D. Cicchetti \& S. L. Toth (Eds.), Child abuse, child development, and social policy (pp. 7-74). Norwood, NJ: Ablex.

Beaver, K. M., Boutwell, B. B., Barnes, J. C., \& Cooper, J. A. (2009). The biosocial underpinnings to adolescent victimization: Results from a longitudinal sample of twins. Youth Violence and Juvenile Justice, 7, 223-238.

Beaver, K. M., Boutwell, B. B., Barnes, J. C., DeLisi, M., \& Vaughn, M. G. (2013). Exploring the genetic origins of adolescent victimization in a longitudinal sample of adoptees. Victims and Offenders, 8, 148-163.

Bifulco, A., Bernazzani, O., Moran, P. M., \& Jacobs, C. (2005). The Childhood Experiences of Care and Abuse Questionnaire (CECA.Q) - Validation in a community series. British Journal of Clinical Psychology, 44, $563-581$.

Bifulco, A., Brown, G. W., \& Harris, T. O. (1994). Childhood Experience of Care and Abuse (CECA): A retrospective interview measure. Journal of Child Psychology and Psychiatry, 35, 1419-1435.

Bifulco, A., Brown, G. W., Lillie, A., \& Jarvis, J. (1997). Memories of childhood neglect and abuse: Corroboration in a series of sisters. Journal of Child Psychology and Psychiatry, 38, 365-374.

Bifulco, A., Brown, G. W., Moran, P., Ball, C., \& Campbell, C. (1998). Predicting depression in women: The role of past and present vulnerability. Psychological Medicine, 28, 39-50.

Bifulco, A., Brown, G. W., Neubauer, A., Moran, P., \& Harris, T. (1994). Childhood Experience of Care and Abuse (CECA) training manual. London: University of London, Royal Holloway College.

Bowes, L., Maughan, B., Ball, H., Shakoor, S., Ouellet-Morin, I., Caspi, A., et al. (2013). Chronic bullying victimization across school transitions: The role of genetic and environmental influences. Development and Psychopathology, 25, 333-346.

Brown, G. W. (1974). Meaning, measurement, and stress of life events. In B. S. Dohrenwend \& B. P. Dohrenwend (Eds.), Stressful life events: Their nature and effects (pp. 217-243). New York: Wiley-Interscience.

Brown, G. W. (1989). Life events and measurement. In G. W. Brown \& T. O. Harris (Eds.), Life events and illness (pp. 3-45). London: Guilford Press.

Brown, G. W., \& Harris, T. O. (1978). Social origins of depression: A study of psychiatric disorder in women. New York: Free Press.

Burt, S. A. (2009). Rethinking environmental contributions to child and adolescent psychopathology: A meta-analysis of shared environmental influences. Psychological Bulletin, 135, 608-637.

Caspi, A., Houts, R., Belsky, D. W., Goldman-Mellor, S., Harrington, H. L., Israel, S., et al. (2014). The "p factor": One general psychopathology factor in the structure of psychiatric disorders? Clinical Psychological Science, 2, 119-137.

Caspi, A., Moffitt, T. E., Thornton, A., Freedman, D., Amell, J. W., Harrington, H., et al. (1996). The life history calendar: A research and clinical assessment method for collecting retrospective event-history data. International Journal of Methods in Psychiatric Research, 6, 101-114.

Clark, L. A., Watson, D., \& Reynolds, S. (1995). Diagnosis and classification of psychopathology: Challenges to the current system and future directions. Annual Review of Psychology, 46, 121-153.

Clemmons, J. C., Walsh, K., DiLillo, D., \& Messman-Moore, T. L. (2007) Unique and combined contributions of multiple child abuse types and abuse severity to adult trauma symptomatology. Child Maltreatment, 12, 172-181

Cicchetti, D., \& Tucker, D. (Eds.) (1994). Neural plasticity, sensitive periods, and psychopathology [Special Issue]. Development and Psychopathology, 6, 531-814.

Cromer, B. (2011). Adolescent development. In R. M. Kliegman, R. E. Behrman, H. B. Jenson, \& B. F. Stanton (Eds.), Nelson textbook of pediatrics (19th ed.). Philadelphia, PA: Saunders Elsevier.

Currie, J., \& Widom, C. S. (2010). Long-term consequences of child abuse and neglect on adult economic well-being. Child Maltreatment, 15, $111-120$.

Danese, A., Moffitt, T. E., Harrington, H., Milne, B. J., Polanczyk, G., Pariante, C. M., et al. (2009). Adverse childhood experiences and adult risk factors for age-related disease: Depression, inflammation, and clustering of metabolic risk markers. Archives of Pediatric and Adolescent Medicine, 163, 1135-1143.

Della Femina, D., Yeager, C. A., \& Lewis, D. C. (1990). Child abuse: Adolescent records versus adult recall. Child Abuse and Neglect, 14, 227-231. 
Dodge, K. A., Bates, J. E., \& Pettit, G. S. (1990). Mechanisms in the cycle of violence. Science, 250, 1678-1683.

Dohrenwend, B. P. (2006). Inventorying stressful life events as risk factors for psychopathology: Toward resolution of the problem of intracategory variability. Psychological Bulletin, 132, 477-495.

Fergusson, D. M., Boden, J. M., \& Horwood, L. J. (2008). Exposure to childhood sexual and physical abuse and adjustment in early adulthood. Child Abuse and Neglect, 32, 607-619.

Finkehor, D., Hamby, S. L., Ormrod, R. K., \& Turner, H. A. (2005). The Juvenile Victimization Questionnaire: Reliability, validity, and national norms. Child Abuse and Neglect, 29, 383-412.

Finkehor, D., Hamby, S. L., Turner, H. A., \& Ormrod, R. K. (2011). The Juvenile Victimization Questionnaire (JVQ-R2) (2nd rev.). Durham, NH: Crimes Against Children Research Center.

Finkelhor, D., Ormrod, R. K., \& Turner, H. A. (2007). Polyvictimization and trauma in a national longitudinal cohort. Development and Psychopathology, 19, 149-166.

Finkelhor, D., Turner, H., Ormrod, R., \& Hamby, S. L. (2009). Violence, abuse, and crime exposure in a national sample of children and youth. $\mathrm{Pe}$ diatrics, 124, 1411-1423.

Fisher, H. L., Bunn, A., Jacobs, C., Moran, P., \& Bifulco, A. (2011). Concordance between mother and offspring retrospective reports of childhood adversity. Child Abuse and Neglect, 35, 117-122.

Fisher, H. L., Jones, P. B., Fearon, P., Craig, T. K., Dazzan, P., Morgan, K., et al. (2010). The varying impact of type, timing and frequency of exposure to childhood adversity on its association with adult psychotic disorder. Psychological Medicine, 40, 1967-1978.

Gilbert, R., Widom, C. S., Browne, K., Fergusson, D., Webb, E., \& Janson, S. (2009). Burden and consequences of child maltreatment in high-income countries. Lancet, 373, 68-81.

Gorman, D. M. (1993). A review of studies comparing checklist and interview methods of data collection in life event research. Behavioral Medicine, 19, 66-73.

Grant, K. E., Compas, B. E., Thurm, A. E., McMahon, S. D., \& Gipson, P. Y. (2004). Stressors and child and adolescent psychopathology: Measurement issues and prospective effects. Journal of Clinical Child and Adolescent Psychology, 33, 412-425.

Groeneveld, L. P., \& Giovannoni, J. M. (1977). Disposition of child abuse and neglect cases. Social Work Research Abstracts, 13, 24-30.

Hamby, S., Finkelhor, D., Ormrod, D., \& Turner, H. (2004). The comprehensive JV administration and scoring manual. Durham, NH: University of New Hampshire, Crimes Against Children Research Centre.

Hardt, J., \& Rutter, M. (2004). Validity of adult retrospective reports of adverse childhood experiences: Review of the evidence. Journal of Child Psychology and Psychiatry, 45, 260-273.

Harris, J. R. (1998). The nurture assumption. New York: Free Press.

Hepp, U., Gamma, A., Milos, G., Eich, D., Ajdacic-Gross, V., Rössler, W., et al. (2006). Inconsistency in reporting potentially traumatic events. British Journal of Psychiatry, 188, 278-283.

Jaffee, S. R., Caspi, A., Moffitt, T. E., Polo-Tomas, M., Price, T. S., \& Taylor, A. (2004). The limits of child effects: Evidence for genetically mediated child effects on corporal punishment but not on physical maltreatment. Developmental Psychology, 40, 1047-1058.

Jaffee, S. R., Caspi, A., Moffitt, T. E., Polo-Tomas, M., \& Taylor, A. (2007). Individual, family, and neighborhood factors distinguish resilient from non-resilient maltreated children: A cumulative stressors model. Child Abuse and Neglect, 31, 231-253.

Jaffee, S. R., Caspi, A., Moffitt, T. E., \& Taylor, A. (2004). Physical maltreatment victim to antisocial child: Evidence of an environmentally mediated process. Journal of Abnormal Psychology, 113, 44-55.

Jones, L. M., Mitchell, K. J., \& Finkelhor, D. (2012). Trends in youth Internet victimization: Findings from three youth Internet safety surveys 20002010. Journal of Adolescent Health, 50, 179-186.

Kendler, K. S., \& Baker, J. H. (2007). Genetic influences on measures of the environment: A systematic review. Psychological Medicine, 37, 615-626.

Lansford, J. E., Dodge, K. A., Pettitm, G. S., Batesm, J. E., Crozier, J., \& Kaplow, J. (2002). Long-term effects of early child physical maltreatment on psychological, behavioral, and academic problems in adolescence: A 12year prospective study. Archives of Pediatrics and Adolescent Medicine, $156,824-830$

Luciana, M. (2013). Adolescent brain development in normality and psychopathology. Development and Psychopathology, 25, 1325-1345.

Matta Oshima, K. M., Jonson-Reid, M., \& Seay, K. D. (2014). The influence of childhood sexual abuse on adolescent outcomes: The roles of gender, poverty, and revictimization. Journal of Child Sexual Abuse, 23, 367386.

Moffitt, T. E., Caspi, A., Krueger, R. F., Magdol, L., Margolin, G., Silva, P. A., et al. (1997). Do partners agree about abuse in their relationship? A psychometric evaluation of interpartner agreement. Psychological Assessment, 9, 47-56.

Moffitt, T. E., \& the E-Risk Study Team. (2002). Teen-aged mothers in contemporary Britain. Journal of Child Psychology and Psychiatry, 43, 723-742.

Moffitt, T. E., \& the Klaus Grawe ThinkTank. (2013). Childhood exposure to violence and lifelong health: Clinical intervention science and stress-biology research join forces. Development and Psychopathology, 25, 1619-1634.

Monroe, S. M. (2008). Modern approaches to conceptualizing and measuring human life stress. Annual Review of Clinical Psychology, 4, 33-52.

Monroe, S. M., \& McQuaid, J. R. (1994). Measuring life stress and assessing its impact on mental health. In W. R. Avison \& I. H. Gotlib (Eds.), Stress and mental health: Contemporary issues and prospects for the future (pp. 43-73). New York: Plenum Press.

Monroe, S. M., \& Reid, M. W. (2008). Gene-environment interactions in depression: Genetic polymorphisms and life stress polyprocedures. Psychologica Science, 19, 947-956.

Neale, M. C., \& Cardon, L. R. (1992). Methodology for genetic studies of twins and families. Dordrecht: Kluwer Academic.

Newman, D. L., Moffitt, T. E., Caspi, A., Magdol, L., Silva, P. A., \& Stanton, W. R. (1996). Psychiatric disorder in a birth cohort of young adults: Prevalence, comorbidity, clinical significance, and new case incidence from ages 11 to 21. Journal of Consulting and Clinical Psychology, 64, 552562.

Norman, R. E., Byambaa, M., De, R., Butchart, A., Scott, J., \& Vos, T. (2012). The long-term health consequences of child physical abuse, emotional abuse, and neglect: A systematic review and meta-analysis. PLOS Medicine, 9, e1001349.

Odgers, C. L., Caspi, A., Bates, C. J., Sampson, R. J., \& Moffitt, T. E. (2012). Systematic social observation of children's neighborhoods using Google Street View: A reliable and cost-effective method. Journal of Child Psychology and Psychiatry, 53, 1009-1017.

Odgers, C. L., Caspi, A., Russell, M. A., Sampson, R. J., Arseneault, L., \& Moffitt, T. E. (2012). Supportive parenting mediates neighborhood socioeconomic disparities in children's antisocial behaviour from ages 5 to 12. Development and Psychopathology, 24, 705-721.

Paykel, E. S. (2001). The evolution of life events research in psychiatry. Journal of Affective Disorders, 62, 141-149.

Pelton, L. H. (1978). Child abuse and neglect: The myth of classlessness American Journal of Orthopsychiatry, 48, 608-617.

Plomin, R. (1994). The nature of nurture. Newbury Park, CA: Sage.

Plomin, R., \& Bergeman, C. S. (1991). The nature of nurture: Genetic influence on "environmental" measures. Behavioral and Brain Sciences, 14 373-386.

Radford, L., Corral, S., Bradley, C., \& Fisher, H. L. (2013). The prevalence and impact of child maltreatment and other types of victimization in the UK: Findings from a population survey of caregivers, children and young people and young adults. Child Abuse and Neglect, 37, 801-813.

Radford, L., Corral, S., Bradley, C., Fisher, H., Bassett, C., Howat, N., et al. (2011). Child abuse and neglect in the UK today. London: NSPCC.

Scarr, S. (1993). Biological and cultural diversity: The legacy of Darwin for development. Child Development, 64, 1333-1353.

Schilling, E. A., Aseltine, R. H., Jr., \& Gore, S. (2008). The impact of cumulative childhood adversity on young adult mental health: Measures, models, and interpretations. Social Science and Medicine, 66, 1140-1151.

Schwarz, N. (2007). Cognitive aspects of survey methodology. Applied Cognitive Psychology, 21, 277-287.

Shakoor, S., Jaffee, S., Andreou, P., Bowes, L., Ambler, A. P., Caspi, A., et al. (2011). Mothers and children as informants of bullying victimisation: Results from an epidemiological cohort of children. Journal of Abnormal Child Psychology, 39, 379-387.

Straus, M. A. (1990). Measuring intrafamily conflict and violence: The Conflict Tactics (CT) scales. In M. A. Straus \& R. G. Gelles (Eds.), Physical violence in American families: Risk factors and adaptations to violence in 8,145 families (pp. 403-424). New Brunswick, NJ: Transaction Press.

Takizawa, R., Maughan, B., \& Arseneault, L. (2014). Adult health outcomes of childhood bullying victimization: Evidence from a five-decade longitudinal British birth cohort. American Journal of Psychiatry, 171, 777-784.

Thornberry, T. P., Ireland, T. O., \& Smith, C. A. (2001). The importance of timing: The varying impact of childhood and adolescent maltreatment on multiple problem outcomes. Development and Psychopathology, 13, 957-979. 
Trouton, A., Spinath, F. M., \& Plomin, R. (2002). Twins Early Development Study (TEDS): A multivariate, longitudinal genetic investigation of language, cognition and behaviour problems in childhood. Twin Research, $38,444-448$.

Trzesniewski, K. H., Moffitt, T. E., Caspi, A., Taylor, A., \& Maughan, B. (2006). Revisiting the association between reading achievement and antisocial behaviour: New evidence of an environmental explanation from a twin study. Child Development, 77, 72-88.

van Dongen, J., Slagboom, P. E., Draisma, H. H. M., Martin, N. G., \& Boomsma, D. I. (2013). The continuing value of twin studies in the omics era. Nature Reviews Genetics, 13, 640-653.

Wertz, J., \& Pariante, C. M. (2014). Invited commentary on . . Psychiatric resilience: Longitudinal twin study. British Journal of Psychiatry, 205, 281-282.
Widom, C. S. (1988). Sampling biases and implications for child abuse research. American Journal of Orthopsychiatry, 58, 260-270.

Widom, C. S., Czaja, S. J., Bentley, T., \& Johnson, M. S. (2012). A prospective investigation of physical health outcomes in abused and neglected children: New findings from a 30-year follow-up. American Journal of Public Health, 102, 1135-1144.

Widom, C. S., Czaja, S. J., \& DuMont, K. A. (2015). Intergenerational transmission of child abuse and neglect: Real or detection bias? Science, 347, $1480-1485$.

Widom, C. S., Czaja, S. J., \& Dutton, M. A. (2008). Childhood victimization and lifetime revictimization. Child Abuse and Neglect, 32, 785-796.

Williams, R. L. (2000). A note on robust variance estimation for cluster-correlated data. Biometrics, 56, 645-646. 